\title{
Depth Perception as a Function of Motion Parallax and Absolute-Distance Information
}

\author{
Mika E. Ono, Josée Rivest, and Hiroshi Ono \\ York University, North York, Ontario, Canada
}

\begin{abstract}
The results of three experiments demonstrated that the visual system calibrates motion parallax according to absolute-distance information in processing depth. The parallax was created by yoking the relative movement of random dots displayed on a cathode-ray tube to the movements of the head. In Experiment 1, at viewing distances of $40 \mathrm{~cm}$ and $80 \mathrm{~cm}$, observers reported the apparent depth produced by motion parallax equivalent to a binocular disparity of $0.47^{\circ}$. The mean apparent depth at $80 \mathrm{~cm}$ was 2.6 times larger than at $40 \mathrm{~cm}$. In Experiment 2, again at viewing distances of $40 \mathrm{~cm}$ and $80 \mathrm{~cm}$, observers adjusted the extent of parallax so that the apparent depth was $7.0 \mathrm{~cm}$. The mean extent of parallax at $80 \mathrm{~cm}$ was $31 \%$ of that at $40 \mathrm{~cm}$. In Experiment 3, distances ranged from $40 \mathrm{~cm}$ to $320 \mathrm{~cm}$, and a wide range of parallax was used. As distance and parallax increased, the perception of a rigid three-dimensional surface was accompanied by rocking motion; perception of depth was replaced by perception of motion in some trials at $320 \mathrm{~cm}$. Moreover, the mean apparent depths were proportional to the viewing distance at $40 \mathrm{~cm}$ and $80 \mathrm{~cm}$ but not at $160 \mathrm{~cm}$ and $320 \mathrm{~cm}$.
\end{abstract}

Motion parallax is defined as the "relative movement of images across the retina resulting from movement of the observer" (Rogers \& Graham, 1979, p. 125). By this definition, motion parallax by itself cannot specify scalar information of depth.' However, with the addition of absolute-distance information (the spatial separation between a point and the observer), motion parallax can specify scalar depth (the spatial separation between two points in the sagittal plane). The central question of this article is: Does the visual system calibrate or scale motion parallax according to absolute-distance information?

To date, no one has raised this question. This is surprising, for motion parallax has been known for approximately five centuries, and was rigorously studied in Germany around the turn of this century (Hell, 1981). Members of the German scientific community became interested in motion parallax in their attempts to determine compensation for the loss of one eye. However, experimental interest was largely focused on the question of whether people can regain depth perception after the loss of one eye comparable to that provided by binocular vision. Interest in motion parallax has been revived in recent years, but the studies are limited to the question of motion parallax as a cue for absolute distance (e.g., Ferris, 1972; Gogel, 1982) or for

This research was supported by Grant A0296 from the Natural Sciences and Engineering Research Council of Canada, by Grant EY05960 from the National Institute of Health, and by the Hospital for Sick Children Foundation.

The authors wish to thank M. Ohmi and S. Saida for designing the apparatus, and S. Anstis, P. Cavanagh, J. Codd, E. González, I. P. Howard, A. Mapp, K. Shimono, M. Steinbach, M. Swanston, and W. J. Tam for helpful comments on an earlier version of this article. We also wish to thank M. Braunstein, W. Epstein, W. C. Gogel, and B. Rogers for their valuable comments during the reviewing process.

Correspondence concerning this article should be addressed to Hiroshi Ono, Department of Psychology, York University, 4700 Keele Street, North York, Ontario, Canada, M3J 1 P3. depth at one viewing distance (e.g., Rogers \& Graham, 1983). See Gibson, Gibson, Smith, and Flock (1959), Nakayama (1985), and Rogers and Graham (1979) for a synopsis of work on motion parallax.

The question of whether motion parallax is calibrated by absolute-distance information is a particularly interesting one because stereoscopic scalar depth results from the processing of binocular disparity and distance information (e.g., Wallach \& Zuckerman, 1963). Moreover, there is a formal similarity between motion parallax and binocular disparity, and many researchers state that the same laws may apply to both (e.g., Barnard \& Thompson, 1980; Gogel, 1978; Graham \& Rogers, 1982). Indeed in 1866, Helmholtz noted:

Since the two eyes occupy positions in space that are not quite the same, the objects in front of us are seen from two slightly different points of view . . . there is the same kind of difference in the images as would be produced by moving in space from one place to the other. (p. 295)

The visual system for motion parallax uses successive information from the relative motion of images; for binocular disparity it uses simultaneous information from fixed disparate images. Both cues, however, consist of the angular separation of corresponding images in the determination of depth.

The inverse square law approximates the relation of disparity to stereoscopic depth and distance (for a derivation, see Ono \& Comerford, 1977):

$$
\delta=\frac{d i}{D^{2}},
$$

where $\delta$ is the binocular disparity (in radians), $d$ is the depth within the stimulus, $D$ is the distance from the observer to the

\footnotetext{
${ }^{1}$ Ono (1970) states that motion-parallax information can lead to scalar depth. In making this statement, he assumed that processing of absolute-distance information is included in the concept of motion parallax.
} 
stimulus, and $i$ is the interocular distance. This equation can be stated in terms of motion parallax by letting $\delta$ be the extent of motion parallax, and $i$ the extent of head movement. In both cases, the inverse square law describes the geometric relations among disparity/motion parallax, depth, and distance. Hence, if the visual system processes information about motion parallax and distance correctly, depth perception will be veridical and depth constancy will follow. For this to occur, the absolutedistance information must be scalar as provided by accommodation, convergence, vertical angle of regard, familiar size cues (see Ono, 1970), and/or a combination of relative distance cues, and additional information such as the observer's translational velocity (see Nakayama, 1985).

Does the visual system calibrate motion parallax according to absolute-distance information in processing depth perception? Experiments 1 and 2 were designed to answer this question. In Experiment 3, we attempted to map the range of depth perception as a function of distance and motion parallax. In all three experiments, the viewing conditions were a normal indoor environment, and we assumed that there was ample absolutedistance information.

\section{Experiment 1}

This experiment is analogous to Holway and Boring's (1941) classic experiment on size perception in which observers were asked to estimate the size of a stimulus at different distances with the visual angle held constant. In our experiment, observers were asked to report the apparent depth within the stimulus at different viewing distances with the extent of retinal motion parallax (proximal motion parallax) held constant. According to the inverse square law, when distance is doubled, apparent depth should quadruple; if parallax were not calibrated according to absolute-distance information, apparent depth should remain the same.

\section{Method}

The display simulated a sinusoidally corrugated three-dimensional surface similar to that of Rogers and Graham (1979). A video camera (Sony AVC-3200) displayed black and white randomly distributed cells providing "random dots" on a TV monitor. The observer's head was supported on a chinrest which could move $30 \mathrm{~cm}$ along a track parallel to the face of the monitor's screen. A potentioneter was used to yoke the horizontal movement of the observer's head to the movement of dots on the screen: The amplitude of the horizontal deflection signal of the monitor was modified by a sinusoidal signal from a function generator (Wavetek model 184) in synchrony with the vertical signal of the monitor. (See Figure 1.) The amplitude of the sinusoidal signal varied with the position of the chinrest so the image on the screen was systematically distorted with the observer's head movement. As the head moved, the horizontal band of dots representing the troughs of the corrugations moved across the screen in the same direction as the obseryer's head movement, while those representing the peaks of the corrugations moved in the opposite direction. The apparent pattern of depth appeared to recede into the monitor set, and not to project out of it. The display was viewed in a room illuminated by fluorescent lights, and care was taken to eliminate reflections of the room lights on the screen.

In the present article, motion parallax is expressed in units of "equivalent disparity" (see Graham \& Rogers, 1982), which allows us to compare it to binocular retinal disparity. Equivalent disparity is calculated by using the successive difference in the visual direction of similar im- ages. In these calculations, a $6.0-\mathrm{cm}$ horizontal movement of the head is analogous to an interocular distance of $6.0 \mathrm{~cm}$ in the calculations of binocular disparity. For Experiment 1, the equivalent disparity was kept constant at $0.47^{\circ}$. This disparity corresponds to peak-to-trough depths of 2.3 and $9.8 \mathrm{~cm}$ at viewing distances of $40 \mathrm{~cm}$ and $80 \mathrm{~cm}$, respectively. ${ }^{2}$

The visible portion of the screen, the peak-to-peak distance of the apparent corrugations, and the random dot density were varied together in such a way that there were two display conditions (large and small) for each viewing distance. For the large display, the visible portion of the screen was $30.0 \mathrm{~cm}$ wide and $28.0 \mathrm{~cm}$ high; the peak-to-peak distance of the corrugations was $18.0 \mathrm{~cm}$; and the random dot density was 8.8 cells/ $\mathrm{cm}^{2}$. For the small display, the width and height of the visible portion of the screen and the peak-to-peak distance of the corrugations were half the large one. The dot density was fourfold. The probability of a cell on display being either black or white was 0.5 .

The four conditions resulting from the combination of the two displays and the two viewing distances provided two pairs of equal distal stimuli and one pair of equal proximal stimuli. The two equal proximal stimuli were equal in terms of the size of the visible portion of the screen, the peak-to-peak distance of the corrugations, and the random dot density. Expressed in angular terms, the visual angle of the screen was $19.8^{\circ} \times 21.0^{\circ}$, the visual angle from peak-to-peak was $12.5^{\circ}$, and there were $17.3 \mathrm{cells} / \mathrm{deg}^{2}$ for both the small display condition at $40 \mathrm{~cm}$ and the large display condition at $80 \mathrm{~cm}$. For the large display condition viewed at $40 \mathrm{~cm}$, the visual angle of the screen was $40.0^{\circ} \times 42.0^{\circ}$, the visual angle from peak-to-peak was $25.0^{\circ}$, and there were $4.3 \mathrm{cells} / \mathrm{deg}^{2}$. For the small display condition viewed at $80 \mathrm{~cm}$, the screen was $10.0^{\circ} \times$ $10.5^{\circ}$, the visual angle from peak-to-peak was $6.3^{\circ}$, and there were 68.9 cells/deg ${ }^{2}$.

Observers were asked to move their heads from side-to-side at a speed and extent that felt comfortable. (In a pilot study, we observed that the extent and speed of head movements did not affect the apparent depth, thus confirming what was found by Rogers \& Graham, 1982.) They manually adjusted the distance between two wooden rods to match the apparent depth between the peaks and troughs of the corrugations. They were encouraged to take as much time as necessary to make their evaluation and were permitted to view the rods while they were moving their head and when they were making their final report. Monocular viewing with the preferred eye was used to eliminate conflicting binocular disparity cues which would indicate a flat surface.

The order of the trials was counterbalanced in two blocks of 16 trials. In each block, four trials were conducted at one distance followed by eight trials at the second distance and ending with four trials at the initial distance. The starting distance alternated between $40 \mathrm{~cm}$ and $80 \mathrm{~cm}$ across observers. In each block, observers made two observations with the large display followed by four observations with each of the small, large, then small displays, ending with two more observations with the large display.

The 12 observers were recruited from the University community. They reported no uncorrected visual anomalies. Two observers saw some rocking motion of the pattern at $80 \mathrm{~cm}$ in the small display. The results of these observers were not included in the analysis involving magnitude of depth, because perceived movement appeared to be at the

\footnotetext{
${ }^{2}$ The exact values of these depths depend on the assumptions one makes. These depths were computed according to the way depth was perceived, that is, receding into the monitor set. They were computed by using the following formula:

$$
d=\frac{D(D+d) \delta}{6}=\frac{\delta D^{2}}{6-\delta D},
$$

where $d$ is the predicted depth, $D$ is the distance from the observer and the stimulus, and $\delta$ is the equivalent disparity. If we had assumed that the peak projects out of the screen and the trough recedes, the values would be different.
} 
expense of apparent depth. The issue of perceived movement will be addressed in Experiment 3 . Seven of the remaining 10 observers were naive as to the purpose of the experiment.

\section{Results and Discussion}

Two sets of analyses were performed on the data. The data for the first analysis were the mean apparent depths obtained for each observer in each condition. The data for the second analysis were the ratios of apparent depth at the far distance to that at the near distance. The $95 \%$-confidence interval $(95 \%-\mathrm{CI})$ for a geometric mean of the ratios was calculated and was used to examine whether the theoretically predicted value was contained in this interval.

The mean apparent depth for each condition is shown in Table 1. A three-way repeated measures analysis of variance with Distance (near and far), Display (large and small), and Observers ( 10 individuals) as factors was performed on the data.

The interaction (Distance $\times$ Display) was statistically significant, $F(1,9)=20.98, p<.01$, but the value of omega square showed that it accounted for only $2 \%$ of the variance. Tukey tests showed that at $40 \mathrm{~cm}$ the mean in the small display condition was greater than that in the large display condition, $q(2$, $9)=6.58, p<.01$. At the $80 \mathrm{~cm}$ distance, the mean difference between the two display conditions was not statistically significant. This interaction will be discussed shortly.

As hypothesized, viewing distance affected apparent depth even though the extent of parallax was constant. The main effect of display was not significant, $F(1,9)=2.60, p>.05$, but the main effect of distance was highly significant, $F(1,9)=30.36$, $p<.001$, and accounted for $69 \%$ of the variance. Distance was by far the most important factor. These results support the idea that the same extent of motion parallax produces different depths at different distances.

According to the inverse square law, for a given degree of parallax, apparent depth should quadruple when distance is doubled. To determine whether the data agreed with the values predicted from the inverse square law, the ratios of apparent depth at the far distance to that at the near distance were computed. These ratios were computed for each observer's data and statistical analyses were performed on their logarithms. The geometric mean was $3.5(95 \%-\mathrm{CI}: 3.0$ to 4.0$)$ for the large display and

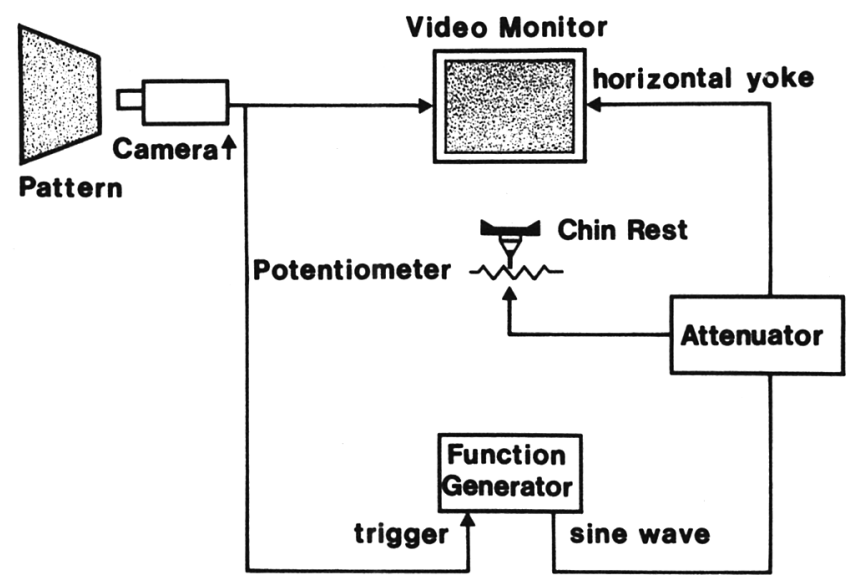

Figure 1. Schematic illustration of the apparatus.
Table 1

Mean Apparent Depth (and SD) in Centimeters for Two Displays and Two Distances in Experiment I

\begin{tabular}{cccccc}
\hline & \multicolumn{4}{c}{ Display } \\
\cline { 2 - 3 } & \multicolumn{2}{c}{ Large } & & \multicolumn{2}{c}{ Small } \\
\cline { 2 - 3 } \cline { 5 - 6 } Distance & $M$ & $S D$ & & $M$ & $S D$ \\
\hline $40 \mathrm{~cm}$ & 2.6 & 0.84 & & 4.0 & 1.30 \\
$80 \mathrm{~cm}$ & 9.0 & 2.28 & & 8.4 & 2.30 \\
\hline
\end{tabular}

2.2 (95\%-CI: 1.9 to 2.5 ) for the small display. The geometric mean of the ratios for the two equal proximal conditions (the small display at the nearest distance and the large display at the farthest distance) was 2.3 (95\%-CI $=2.0$ to 2.7$)$. Only the confidence interval for the large display contained the predicted value of 4 .

An analysis of variance was performed on the logarithms of the ratios of apparent depth at the two distances as a function of display (large display conditions, small display conditions, and equal proximal conditions). The effect of display was statistically significant, $F(2,9)=5.64, p<.05$. Tukey tests showed that the geometric mean of the ratio with the largest display was statistically larger than the mean of the ratio of the smallest display, $q(2,18)=11.55, p<.01$, and from the mean of the ratio of the equal proximal conditions, $q(2,18)=10.00, p<$ .01 . There was no significant difference between the mean for the small display and that for the displays which produced the same proximal stimulation, $q(2,18)=1.73, p>.05$. The first difference reflects the statistical interaction noted in the earlier analysis (i.e., Distance $X$ Display interaction). We do not have an explanation for the large ratio of apparent depth with the large display. This difference was not reproduced in Experiment 2 ; thus, it may be specific to the task used in this experiment.

The fact that motion parallax produced different depths at different distances supports the idea that the visual system calibrates motion parallax according to absolute-distance information. Although the ratios obtained were less than the 4:1 predicted from the inverse square law, they are a good deal larger than a ratio of $1: 1$, which would indicate that the visual system does not calibrate motion parallax according to absolute-distance information.

\section{Experiment 2}

This experiment also tested the inverse square law for apparent depth produced by motion parallax. If apparent depth follows the inverse square law, depth constancy also exists (see Ono \& Comerford, 1977). Observers were asked to adjust the extent of motion parallax to hold the magnitude of apparent depth constant at two different distances. When the distance is doubled, the adjusted extent of motion parallax on the retina at the far distance should be $25 \%$ of that at the nearer distance. If parallax is not calibrated according to absolute-distance information, the adjusted extent of motion parallax should remain the same. 
Table 2

Mean Parallax Settings (and SD) in Degrees for Two Displays and Two Distances in Experiment 2

\begin{tabular}{cccccc} 
& \multicolumn{4}{c}{ Display } \\
\cline { 2 - 4 } & \multicolumn{2}{c}{ Large } & & \multicolumn{2}{c}{ Small } \\
\cline { 2 - 3 } \cline { 5 - 6 } Distance & $M$ & $S D$ & & $M$ & $S D$ \\
\hline $40 \mathrm{~cm}$ & 1.23 & 0.25 & & 1.07 & 0.27 \\
$80 \mathrm{~cm}$ & 0.38 & 0.11 & & 0.33 & 0.11 \\
\hline
\end{tabular}

\section{Method}

The apparatus, viewing distances, counterbalancing, and controls were the same as in Experiment 1. At the two viewing distances, observers turned a knob which controlled the extent of movement on the screen. They adjusted the amount of motion parallax until the depth of the corrugations appeared equal to a 7.0-cm block of wood which they held in their hand. The $7.0 \mathrm{~cm}$ of depth at $40 \mathrm{~cm}$ and $80 \mathrm{~cm}$ corresponds to the equivalent disparities of $1.28^{\circ}$ and $0.34^{\circ}$, respectively. Again, observers viewed the display with their preferred eye and were encouraged to take their time making their judgements. They were permitted to view the block of wood while they were moving their head.

The 12 observers were the same as in Experiment 1 . Three observers saw a rocking motion of the pattern in some of the trials at $80 \mathrm{~cm}$ in the small display condition within the range of their depth settings. The data from these observers were not included in the analysis involving magnitude of depth. Six of the remaining 9 observers were naive regarding the purpose of the experiment.

\section{Results and Discussion}

A three-way repeated measures analysis of variance with the same factors as in Experiment 1 was performed on the mean parallax settings. (See Table 2 for mean parallax settings.) The analysis showed that the interaction (Distance $\times$ Display) was not significant, $F(1,8)=4.30, p>.05$. The main effect of distance was statistically significant, $F(1,8)=183.48, p<.001$, and accounted for $80 \%$ of the variance. All observers gave higher mean parallax settings at the close distance.

In addition to the main effect of distance, the main effect of display was significant, $F(1,8)=6.2, p<.05$; however, it accounted for only $1 \%$ of the variance. On average, the adjusted parallax was larger with the large display than with the small display $\left(0.81^{\circ}\right.$ and $\left.0.70^{\circ}\right)$. This significant main effect of display and the nonsignificant interaction (Distance $\times$ Display) contrast with the results of Experiment 1, in which the main effect of display was not significant, but the interaction (Distance $X$ Display) was significant. Thus, these effects may be specific to the different tasks of the two experiments.

The ratios of the parallax settings were computed as in Experiment 1 . The mean of the ratios was 0.30 (95\%-Cl: 0.26 to 0.36 ) for the large display conditions; 0.29 (95\%-CI: 0.25 to 0.35 ) for the small display conditions; and 0.35 (95\%-CI: 0.29 to 0.41 ) for the equal proximal conditions. Two of these confidence intervals did not contain the predicted value of 0.25 . A two-way analysis of variance computed on the logarithms of the ratios showed no significant differences among the three conditions of display, $F(2,16)=0.43, p>.05$.

The basic results of Experiment 2 were the same as those of
Experiment 1. Although the obtained ratios did not agree exactly with the predicted ratio of 0.25 , they were considerably smaller than a ratio of unity, which would indicate that motion parallax is not calibrated according to absolute-distance information. Therefore, the results lend further support to the idea that the visual system calibrates motion parallax according to absolute-distance information. The reasons for the discrepancies between the predicted and obtained values of apparent depth found in Experiment 1 and 2 may become clear when convergence state and apparent distance are measured or manipulated to examine their effect on apparent depth as done in studies of stereoscopic depth constancy (e.g., Fried, 1974; Gogel, 1972, 1978; O'Leary \& Wallach, 1980; Wallach, Gillam, \& Cardillo, 1979).

\section{Experiment 3}

Experiments 1 and 2 showed that the visual system calibrates the extent of motion parallax according to absolute-distance information for depth perception. Experiment 3 was designed to map the range of this process. In this experiment three distal parallaxes were held constant over viewing distances. The approach is analogous to the one taken in Wallach and Zuckerman's (1963) study of stereoscopic depth constancy. In their stimulus arrangement, as in ours, no constancy would be reflected by the apparent depth being halved when the distance doubles, and complete constancy (the inverse square law) would be reflected by apparent depth being doubled. The first prediction derives from the fact that keeping the same extent of movement on the display while doubling the distance would halve the extent of movement on a retina; thus, the parallax or disparity would be halved as the distance doubles. The second prediction comes from substituting 0.5 for parallax, or disparity, in the formula presented in the introduction.

\section{Method}

The same apparatus was used as in Experiments 1 and 2, but observers now viewed the stimulus at four distances: $40 \mathrm{~cm}, 80 \mathrm{~cm}, 160 \mathrm{~cm}$, and $320 \mathrm{~cm}$. Three different distal parallax settings were used. At 40 $\mathrm{cm}$, the extents of proximal motion parallax were equivalent to $0.47^{\circ}$, $0.94^{\circ}$, and $1.88^{\circ}$ of equivalent disparity. For these distal parallaxes, the proximal parallaxes halved whenever distance doubled. Only the large display condition was employed (see Experiment 1 for description). Thus, as distance doubled, the proximal size (height and width) of the visible portion of the screen and the distance from peak-to-peak of the corrugation were halved, and the dot density was quadrupled.

For each trial, observers were asked to indicate whether they saw (a) depth without motion-an apparent rigid three-dimensional surface without movement; (b) depth with rocking motion-an apparent rotation of a corrugated surface along a vertical axis ${ }^{3}$; or (c) no depth at all, only motion. If (a) depth without motion or (b) depth and rocking motion was perceived, observers adjusted the spatial separation between two rods to match the apparent depth of the display, as in Experiment 1. The criterion for distinguishing between (a) and (b) and between (b) and (c) probably varied within and among observers. For example, 1

\footnotetext{
${ }^{3}$ The authors and their colleagues made observations on the rocking motion after the study. In the experimental setting, the axis is frequently located at the far surface (trough), sometimes midway between the far surface (trough) and the near surface (peak) but almost never at the near surface (peak).
} 
Table 3

Number of Observers Who Saw Only Motion in at Least One of the Trials as a Function of Distance and Distal Parallax in Experiment 3

\begin{tabular}{lcccc}
\hline & \multicolumn{4}{c}{ Distance } \\
\cline { 2 - 5 } $\begin{array}{c}\text { Distal } \\
\text { parallax }\end{array}$ & $40 \mathrm{~cm}$ & $80 \mathrm{~cm}$ & $160 \mathrm{~cm}$ & $320 \mathrm{~cm}$ \\
\hline Small & 0 & 0 & 1 & 6 \\
Medium & 0 & 0 & 1 & 5 \\
Large & 0 & 0 & 1 & 3 \\
\hline
\end{tabular}

Note. $n=12$.

observer claimed always to see some rocking motion in Experiment 3, although he did not report this in Experiments I and 2. Although the boundaries between categories may not be clear, most observers had no problem distinguishing among them.

There were two blocks of 24 trials, with a break after the first block. Within each block, judgments were made at four distances and for three distal parallax settings. Two trials for each of the distal parallax settings were included at each distance. The order of the trials was randomized for each block. The same 12 observers participated in Experiment 3 as in the other two experiments. Seven of the 12 observers were naive concerning the purpose of the experiment.

\section{Results and Discussion}

This section is divided into three parts. The first part reports and discusses observers' seeing depth and/or motion. The second part reports the magnitudes of depth with rocking motion and discusses them in terms of depth perception being at the expense of motion perception or vice versa. The third part reports the magnitudes of depth without motion and discusses them in terms of the predictions from the inverse square law.

Seeing depth and/or motion. With our experimental stimuli, depth perception produced by motion parallax had a limited range: As viewing distance increased, more observers lost depth perception altogether and perceived only motion (see Table 3 ). Moreover, more observers saw a rocking motion and fewer observers saw depth without motion as the extent of motion parallax and/or distance increased. The number of these observers for each condition is presented in Table 4. These results, when considered with those from Experiments 1 and 2, suggest that whether the observers perceive depth and/or motion depends on individuals, distal parallax, and viewing distance.

Individuals differed considerably in their perception of depth and/or motion. For example, 1 observer lost depth entirely in most of the trials in the 80-cm small display condition of Experiments 1 and 2, where most observers saw no motion at all. In Experiment 3 another observer almost never saw motion, even at $320 \mathrm{~cm}$, where most of the other observers were seeing motion in at least one of the trials.

Despite the large individual differences, the results suggest that an upper threshold exists for seeing depth as a function of distal parallax. This is suggested by the tendency at each distance for the frequency of "rocking motion" to increase as the distal parallax increased. Whether an upper threshold can be specified by a single angular unit for different distances as is done for retinal disparity may be problematical as is implied by the following discussion.
In terms of retinal stimulation, the size of the visible portion of the screen, the separation between the peaks of the apparent corrugations, and the dot density varied with viewing distances in Experiment 3. Although all these variables may have contributed to changes in depth or motion perception, viewing distance is clearly the critical factor in determining whether the observer sees depth and/or motion. The proximal stimulus in terms of size of the visible portion of the screen, the peak separation, and the dot density for the small display condition at $80 \mathrm{~cm}$ in Experiment 1 were equivalent to the large distal parallax condition at $160 \mathrm{~cm}$ in Experiment 3 . Even though only the viewing distances varied, 10 out of 12 observers reported seeing depth without rocking motion at $80 \mathrm{~cm}$, whereas only 1 out of 12 reported this in every trial at $160 \mathrm{~cm}$. We do not think this difference was due to "practice" or "learning," because both the proportion of observers reporting depth without motion and the mean apparent depths were very similar in the identical conditions of Experiments 3 and 1. To be more specific, the results from the 40-cm small distal parallax and the $80-\mathrm{cm}$ medium distal parallax conditions were the same as the results from the $40-\mathrm{cm}$ large display and the $80-\mathrm{cm}$ large display conditions, respectively.

In summary, the results suggest that the limit of effective motion parallax cannot be specified simply in terms of proximal parallax when absolute-distance information is available to the visual system.

Depth with rocking motion. The lower mean apparent depth when rocking motion was also perceived suggests a trade-off between motion and depth perception. The mean apparent depths when observers saw only depth and those when they saw depth with rocking motion are presented in Table 4. (There are a few instances in Table 4 where the apparent depth is greater for the depth with rocking motion, but all of them involve a small number of observers and cannot be considered to be reliable.) Although Experiment 3 was not designed to examine the deter-

Table 4

Mean Apparent Depth (in cm) and Numbers of Observers (No.) for Two Different Percepts as a Function of Distance and Distal Parallax in Experiment 3

\begin{tabular}{|c|c|c|c|c|c|c|c|c|}
\hline \multirow{3}{*}{$\begin{array}{c}\text { Distal } \\
\text { parallax }\end{array}$} & \multicolumn{8}{|c|}{ Distance } \\
\hline & \multicolumn{2}{|c|}{$40 \mathrm{~cm}$} & \multicolumn{2}{|c|}{$80 \mathrm{~cm}$} & \multicolumn{2}{|c|}{$160 \mathrm{~cm}$} & \multicolumn{2}{|c|}{$320 \mathrm{~cm}$} \\
\hline & $M$ & No. & $M$ & No. & $M$ & No. & $M$ & No. \\
\hline \multicolumn{9}{|c|}{ Depth without motion } \\
\hline Small & 2.8 & 11 & 5.6 & 11 & 8.0 & 9 & 12.7 & 5 \\
\hline Medium & 4.8 & 11 & 8.4 & 11 & 11.4 & 8 & 15.6 & 5 \\
\hline Large & 8.9 & 10 & 12.2 & 7 & 17.7 & 4 & 20.1 & 2 \\
\hline \multicolumn{9}{|c|}{ Depth with motion } \\
\hline Small & 3.2 & 1 & 4.7 & 1 & 5.1 & 5 & 7.9 & 7 \\
\hline Medium & 5.3 & $i$ & 6.5 & 3 & 8.2 & 6 & 9.9 & 9 \\
\hline Large & 9.1 & 4 & 10.3 & 8 & 13.3 & 10 & 15.3 & 11 \\
\hline
\end{tabular}

Note. Some observers reported the two different percepts in different trials. These observers are represented in two categories of percept. Therefore, the sums of the number of observers in some conditions are greater than 12 . 
minants of rocking motion and the apparent depth associated with it, the results are discussed because of their theoretical implications. The following are two different theoretical frameworks in which our findings can be considered.

The trade-off we found is consistent with the findings by Gogel (e.g., 1980, 1981) and his analysis of apparent motion concomitant with head movement. According to his analysis, the extent of apparent depth and that of apparent motion is a function of perceived distance and "pivot distance" of points in space. (See e.g., Gogel, 1980, for details.) When depth with rocking motion is seen with our display, the motion parallax is partially effective. When no depth is seen, motion parallax is totally ineffective. In this theoretical framework a question remains: Why does the effectiveness of parallax decrease as a function of viewing distance?

Although this analysis describes the trade-off between apparent motion and apparent depth, it does not distinguish between the percept of "rocking motion"- the perceived rigid three-dimensional corrugated surface rotating on a vertical axis-and a percept of a surface undergoing a shearing transformation or deformation. Given that the percept is that of a rigid surface in motion, we can consider our stimulus at $160 \mathrm{~cm}$ or $320 \mathrm{~cm}$ as simulating the conditions in which direction or location constancy fails. This consideration suggests that Wallach's theoretical framework (see Wallach, 1985, for a synopsis) can be applied to the rocking motion. Under this analysis rocking motion represents a percept outside the "immobility range" where the proprioceptive information from the head movement compensates for the retinal motion. The unanswered question becomes: Why does the compensation fail as the viewing distance increases?

Depth without motion. An analysis of variance for the magnitude of apparent depth was performed on a subset of the data taken from the conditions in which 8 observers saw depth without motion in at least one of the trials. (See Figure 2 for these results.) The basic unit of the analysis was the mean of apparent depth computed for each condition and for each observer, and the three factors were: Distance $(40 \mathrm{~cm}, 80 \mathrm{~cm}, 160 \mathrm{~cm})$, Distal Parallax (small and medium) and Observers ( 8 individuals). The Distance $\times$ Distal Parallax interaction was not significant, $F(2,14)=2.59, p>.05$. The main effect of distance, however, was statistically significant, $F(2,14)=31.17, p<.001$, and accounted for $47 \%$ of the variance. The main effect of distal parallax was also significant, $F(1,7)=65.86, p<.001$, and accounted for $11 \%$ of the variance.

The geometric means of apparent depth ratios for the $80 \mathrm{~cm}$ to $-40 \mathrm{~cm}$ distances were $2.2(95 \%-\mathrm{Cl}: 1.7$ to 2.7$)$ and $1.9(95 \%$ CI: 1.6 to 2.3 ) for the small and medium distal parallax conditions, respectively. These ratios indicate almost complete depth constancy, as the confidence intervals of their corresponding geometrical mean included the value of 2.0 predicted from the inverse square law. This agreement contrasts with the poorer agreement found in Experiments 1 and 2, but the data are similar to those from Wallach and Zuckerman's (1963) study on stereoscopic depth constancy. In their experiment, the ratio of the arithmetic mean of apparent depth from the $46 \mathrm{~cm}$ and 92 $\mathrm{cm}$ distance was 1.7 (no constancy would be denoted by 0.5 ). ${ }^{4}$

For viewing distances of $160 \mathrm{~cm}$ to $80 \mathrm{~cm}$, the results agree less with the values predicted from the inverse square law. The ratios of mean apparent depth were 1.5 (95\%-CI: 1.3 to 1.9$)$ in the small distal parallax condition and $1.3(95 \%$-CI: 1.1 to 1.5$)$

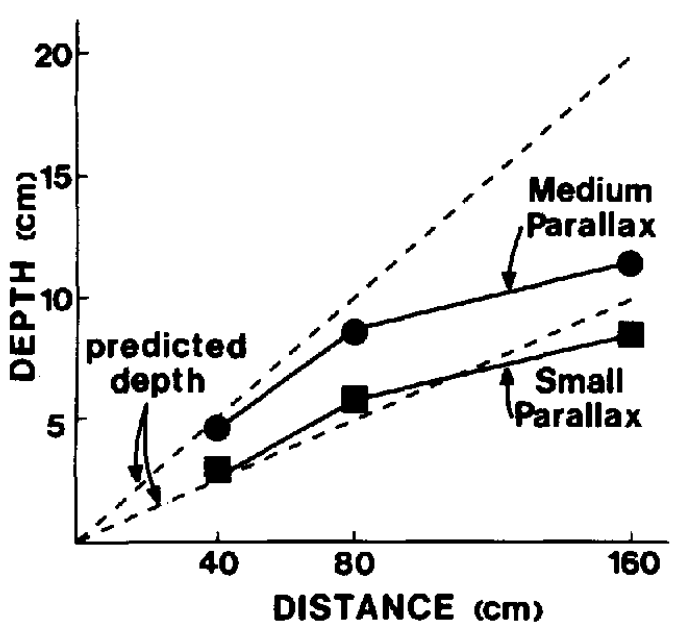

Figure 2. Mean predicted and observed apparent depth (in $\mathrm{cm}$ ) as a function of distance for two distal parallaxes in Experiment 3. $(n=8)$

in the medium distal parallax condition. Since many observers lost depth and/or saw rocking motion at $160 \mathrm{~cm}$ and $320 \mathrm{~cm}$ and at the large distal parallax condition, the number of observations was too small for this set of ratios to be considered reliable.

An analysis of variance for the logarithms of the ratios was performed for the conditions where there were at least eight means. The analysis included three factors: Relative Distances (ratio of apparent depth at $80 \mathrm{~cm}$ to that at $40 \mathrm{~cm}$, and at 160 $\mathrm{cm}$ over that at $80 \mathrm{~cm}$ ), Distal Parallax (small and medium), and Observers ( 8 individuals). The main effect of relative distances was significant, $F(1,7)=8.52, p<.05$; the geometric mean of the ratios from the distances of $160 \mathrm{~cm}$ and $80 \mathrm{~cm}$ (1.4) was significantly smaller than the mean from $80 \mathrm{~cm}$ and $40 \mathrm{~cm}$ (2.0). The main effect of distal parallax was also significant, $F(1$, 7) $=7.00, p<.05$. The mean of the ratios for the medium distal parallax (1.6) was significantly smaller than the mean for the small distal parallax (1.8). These statistically significant differences confirm what is apparent from examining the geometric means for each condition separately. As the distance increases, the ratios deviate below the predicted value.

In summary, even though the degree of agreement between the predicted and obtained values differed from those of Experiments 1 and 2 , the conclusion to be made regarding the calibration of motion parallax according to absolute-distance information is the same. Moreover, motion parallax was not effective for depth perception as distance and parallax increased. Its not being effective at far distances runs counter to most textbook illustrations of motion parallax in which far viewing distances are depicted.

\footnotetext{
${ }^{4}$ Wallach and Zuckerman (1963) reported a ratio of 0.6 for the apparent depth of near-to-far distances. We reported the reciprocal of their ratio in order to be consistent with the analysis of Experiments 1 and 2. In our experiment, the ratios of the arithmetic means of apparent depth for the $40 \mathrm{~cm}$ to $80 \mathrm{~cm}$ distances were 0.4 and 0.5 in the small and medium distal parallax conditions, respectively. They are very close to that found by Wallach and Zuckerman (1963).
} 


\section{General Discussion}

The results from all three experiments clearly show that depth perception arising from motion parallax is contingent upon distance information. However, the magnitudes of apparent depth deviated from the values predicted from the inverse square law in many conditions. Logically, a discrepancy between theoretical and obtained values can be explained by misregistration (or misperception) of distance information, motion-parallax information, or head- (and eye-) movement information. Such discrepancy in stereopsis can also be explained by three corresponding factors, namely, that of distance, disparity, or interocular distance. However, theoretical considerations are given only to the misregistration of distance information (see Ono \& Comerford, 1977), perhaps because the misregistrations of disparity and interocular distance are difficult to conceive. In contrast, misregistration of these three pieces of information can be conceived for motion parallax, and future empirical studies should deal with all three.

Our study suggests many similarities between stereopsis and motion parallax as cues for depth perception. As with the apparent depth produced by retinal disparity, the inverse square law very roughly described the apparent depth produced by motion parallax at near distances. Also, as with the apparent depth produced by retinal disparity, there are limits to depth perception produced by motion parallax. Large extents of motion parallax do not produce depth, and no depth emerges for stereograms with large disparities. Furthermore, the perception of motion with, or instead of, depth produced by motion parallax is analogous to the perception of diplopia with, or instead of, stereoscopic depth. The apparent depth with rocking motion is analogous to apparent depth with diplopia, namely, patent stereopsis. (See Ogle, 1962, for a discussion of patent stereopsis.)

Nonetheless, our study also suggests an important difference between disparity and motion parallax. The range of viewing distances within which depth perception occurs appears to be much smaller for motion parallax. In our study, depth perception usually shifted into motion perception as viewing distance increased. The issue in stereoscopic depth perception has not been whether depth perception occurs at far distances, but whether depth perception follows the inverse square law at far distances (see Cormack, 1984; Ono \& Comerford, 1977). Cormack (1984) conducted a study of stereoscopic depth at distances up to $7.8 \mathrm{~km}$ without diplopia's being reported. There seems to be a different range of depth perception for the two cues; in our experiment motion parallax was effective only at near distances.

\section{References}

Barnard, S. T., \& Thompson, W. B. (1980). Disparity analysis of images. IEEE Transactions on Pattern Analysis and Machine Intelligence, PAM1-2, 333-340.

Cormack, R. H. (1984). Stereoscopic depth perception at far viewing distances. Perception \& Psychophysics, 35, 423-428.

Ferris, S. H. (1972). Motion parallax and absolute distance. Journal of Experimental Psychology, 95, 258-263.
Fried, A. H. (1974). Convergence as a cue to distance. (Doctoral dissertation, New School for Social Research, 1973). Dissertation Abstracts International, 3, 3247B (University Microfilms No. 74-146).

Gibson, E. J., Gibson, J. J., Smith, O. W., \& Flock, H. (1959). Motion parallax as a determinant of perceived depth. Journal of Experimental Psychology, 58, 40-51.

Gogel, W. C. (1972). Scalar perceptions with binocular cues of distance. American Journal of Psychology, 85, 477-498.

Gogel, W. C. (1978). Size, distance and depth perception. In E. C. Carterette \& M. P. Friedman (Eds.), Handbook of Perception (Vol. 9, pp. 305-308). New York: Academic Press.

Gogel, W. C. (1980). The sensing of retinal motion. Perception \& Psychophysics, 28, 155-163.

Gogel, W. C. (1981). Perceived depth is a necessary factor in apparent motion concomitant with head motion: A reply of Shebilske and Proffitt. Perception \& Psychophysics, 29, 173-177.

Gogel, W. C. (1982). Analysis of the perception of motion concomitant with a lateral motion of the head. Perception \& Psychophysics, 32, 241-250.

Graham, M., \& Rogers, B. (1982). Simultaneous and successive contrast effects in the perception of depth from motion-parallax and stereoscopic information. Perception, 11, 247-262.

Hell, W. (1981). Research on monocular depth perception between 1884 and 1914: Influence of the German Unfallversicherungsgesetz [Accident Insurance Law] and of the jurisdiction of the Reichsversicherungsamt [German Insurance Board]. Perception, 10, 683-694.

Helmholtz, H., von. (1962). Helmholtz's treatise on physiological optics: Vol. 3. (3rd ed.). (J. P. C. Southall, Ed. \& Trans. of 1910 ed.). New York: Dover.

Holway, A. H., \& Boring, E. G. (1941). Determinants of apparent visual size with distance variant. American Journal of Psychology, 54, 21 37.

Nakayama, K. (1985). Biological image motion processing: A review. Vision Research, 25, 625-660.

Ogle, K. N. (1962). On limits of stereoscopic vision. Journal of Experimental Psychology, 44, 253-259.

O'Leary, A., \& Wallach, H. (1980). Adaptation in stereoscopic depth constancy. Perception \& Psychophysics, 25, 403-408.

Ono, H. (1970). Some thoughts on different perceptual tasks related to size and distance. Human Space Perception [Psychonomic Monograph Supplements], 3 (13, Whole No. 45), 143-151.

Ono, H., \& Comerford, J. (1977). Stereoscopic depth constancy. In W. Epstein (Ed.), Stability and constancy in visual perception mechanisms \& processes (pp. 91-128). New York: Wiley.

Rogers, B., \& Graham, M. (1979). Motion parallax as an independent cue for depth perception. Perception, 8, 125-134.

Rogers, B., \& Graham, M. (1982). Similarities between motion parallax and stereopsis in human depth perception. Vision Research, 22, 261270.

Rogers, B., \& Graham, M. (1983). Anisotropies in the perception of three-dimensional surfaces. Science, 221, 1409-1411.

Wallach, H. (1985). Perceiving a stable environment. Scientific American, 252, 118-124.

Wallach, H., Gillam, B., \& Cardillo, L. (1979). Some consequences of stereoscopic depth constancy. Perception \& Psychophysics, 26, 235240.

Wallach, H., \& Zuckerman, C. (1963). The constancy of stereoscopic depth. The American Journal of Psychology, 76, 404-412.

Received October 28, 1985

Revision received February 20, 1986 